\title{
Probing Globular Cluster Formation in Low Metallicity Dwarf Galaxies
}

\author{
Kelsey E. Johnson ${ }^{1,2}$, Leslie K. Hunt ${ }^{3}$, and Amy E. Reines ${ }^{1}$ \\ ${ }^{1}$ Astronomy Department, University of Virginia, P.O. Box 400325, \\ Charlottesville, VA, 22904, USA \\ email: kej7a@virginia.edu \\ ${ }^{2}$ The National Radio Astronomy Observatory, 520 Edgemont Road, \\ Charlottesville, VA 22903, USA \\ ${ }^{3}$ INAF-Istituto di Radioastronomia-Sez. Firenze, L.go, Fermi 5, I-50125 Firenze, Italy
}

\begin{abstract}
The ubiquitous presence of globular clusters around massive galaxies today suggests that these extreme star clusters must have been formed prolifically in the earlier universe in low-metallicity galaxies. Numerous adolescent and massive star clusters are already known to be present in a variety of galaxies in the local universe; however most of these systems have metallicities of $12+\log (\mathrm{O} / \mathrm{H})>8$, and are thus not representative of the galaxies in which today's ancient globular clusters were formed. In order to better understand the formation and evolution of these massive clusters in environments with few heavy elements, we have targeted several low-metallicity dwarf galaxies with radio observations, searching for newlyformed massive star clusters still embedded in their birth material. The galaxies in this initial study are HS 0822+3542, UGC 4483, Pox 186, and SBS 0335-052, all of which have metallicities of $12+\log (\mathrm{O} / \mathrm{H})<7.75$. While no thermal radio sources, indicative of natal massive star clusters, are found in three of the four galaxies, SBS 0335-052 hosts two such objects, which are incredibly luminous. The radio spectral energy distributions of these intense star-forming regions in SBS 0335-052 suggest the presence of $\sim 12,000$ equivalent O-type stars, and the implied star formation rate is nearing the maximum starburst intensity limit.
\end{abstract}

Keywords. galaxies: star clusters, galaxies: starburst, HII regions, stars: formation

\section{Introduction}

Ancient globular clusters are ubiquitous around massive galaxies in the relatively nearby universe (e.g. Harris 1991; Brodie \& Strader 2006), providing an important fossil record of conditions in the earlier universe. In fact, given the likely rate of mortality for these massive clusters is $\gtrsim 90 \%$ (Fall \& Zhang 2001; Whitmore et al. 2007), globular clusters must have been formed prolifically during this epoch. However, until the launch of the Hubble Space Telescope, little was known about the formation and early evolution of these extreme clusters.

With the availability of high spatial resolution imaging, large numbers of massive and dense young blue clusters were found in a variety of galaxies. These adolescent massive clusters were dubbed "super star clusters" (SSCs), and studying them became a bit of a cottage industry. Over the following years, strong lines of evidence suggested that many SSCs will, in fact, evolve into globular clusters (e.g. Whitmore 2003). However, a critical issue linking the present-day SSCs and the ancient globular clusters remains: we have not observationally constrained how the low metal abundance in the early universe affected the formation of massive star clusters. As discussed elsewhere in these proceedings, metallicity can affect star formation and evolution in a variety of ways, including the hardness of the stellar spectra, cooling and pressure in the natal material, and dust 
formation (Schaerer 2002; Smith, Norris, \& Crowther 2002; Tumlinson, Venkatesan, \& Shull 2004; Bate 2005).

One way to approach this problem and derive observational constraints on the effect that low metallicity has on the formation of massive star clusters is to study SSC formation that is taking place in low-metallicity environments in the relatively nearby universe.

\section{Embedded Massive Star Clusters Known in Dwarf Galaxies}

By using sensitive radio observations, a number of dwarf galaxies have been found to host natal SSCs, including He 2-10, NGC 5253, IC 4662, II ZW 40, NGC 4490, NGC 4449, NGC 3125, NGC 2573, NGC 4214, and Haro 3 (Turner et al. 2000; Beck et al. 2000; Johnson \& Kobulnicky 2003; Johnson et al. 2004; Johnson et al. 2003; Reines, Johnson, \& Goss 2008; Johnson et al. in prep.; Aversa et al. in prep.). A few of these galaxies have truly impressive populations of natal clusters; in particular, He 2-10 has at least four massive embedded star clusters that (in sum) account for nearly all of thermal-infrared emission from that galaxy (Vacca, Johnson, \& Conti 2002). However, the problem is that despite being dwarfs, these galaxies do not have particularly low metallicities; none of the objects listed above has a metallicity lower than $12+\log (\mathrm{O} / \mathrm{H}) \sim 8.0$.

\section{The Low-Metallicity Sample}

In order to investigate massive star cluster formation in galaxies with even lower metallicities, we have used the Very Large Array to obtain radio observations of four dwarf galaxies with metallicities of $12+\log (\mathrm{O} / \mathrm{H})<7.75$. The galaxies in this sample are HS 0822+3542, UGC 4483, Pox 186, and SBS 0335-052. The observations were carried out at several wavelengths ranging from $1.3 \mathrm{~cm}$ to $6 \mathrm{~cm}$ in order to detect the characteristic thermal free-free emission that originates in the dense regions of ionized gas surrounding newly formed clusters. Down to relatively stringent detection thresholds, no embedded clusters were detected in HS $0822+3542$, UGC 4483, or Pox 186. Because of the sensitivity of these observations, the upper limits on possible natal clusters that could be present but not detected is significant. In these cases, the maximum masses of natal clusters that the three galaxies with non-detections could host ranges from $\sim 8 \times 10^{3} M_{\odot}$ to $\sim 4 \times 10^{4} M_{\odot}$, all of which are smaller than the stellar mass of the R136 cluster in the 30 Dor region of $\sim 6 \times 10^{4} M_{\odot}$ (Hunter et al. 1995, extrapolated down to $\left.0.1 M_{\odot}\right)$. In other words, if natal clusters with masses similar to R136 existed in these three galaxies, they would have been detected. Fortunately, the story does not end there.

\section{The Incredible Natal Clusters in SBS 0335-052}

In contrast to the other three galaxies in the sample, SBS 0335-0552 hosts extremely luminous thermal radio sources in the southern region of the galaxy (Figure 1). The ionizing flux inferred for the entire radio region suggests the equivalent of $\sim 12,000 \mathrm{O}$ type stars are present in the combined radio sources, and the associated instantaneous star formation rate is a remarkable $\sim 23 M_{\odot} \mathrm{yr}^{-1} \mathrm{Mpc}^{-1}$. A comparison between the radio emission from the compact sources and the entire region suggests that up to $\sim 50 \%$ of the ionizing flux could be leaking from the compact HII regions. This high percentage of escaping flux is consistent with the results of Reines et al. (2008), which suggest that the interstellar medium surrounding the natal clusters in SBS 0335-052 is porous and clumpy. Model HII region fits to the radio data for these sources indicate that the average density 


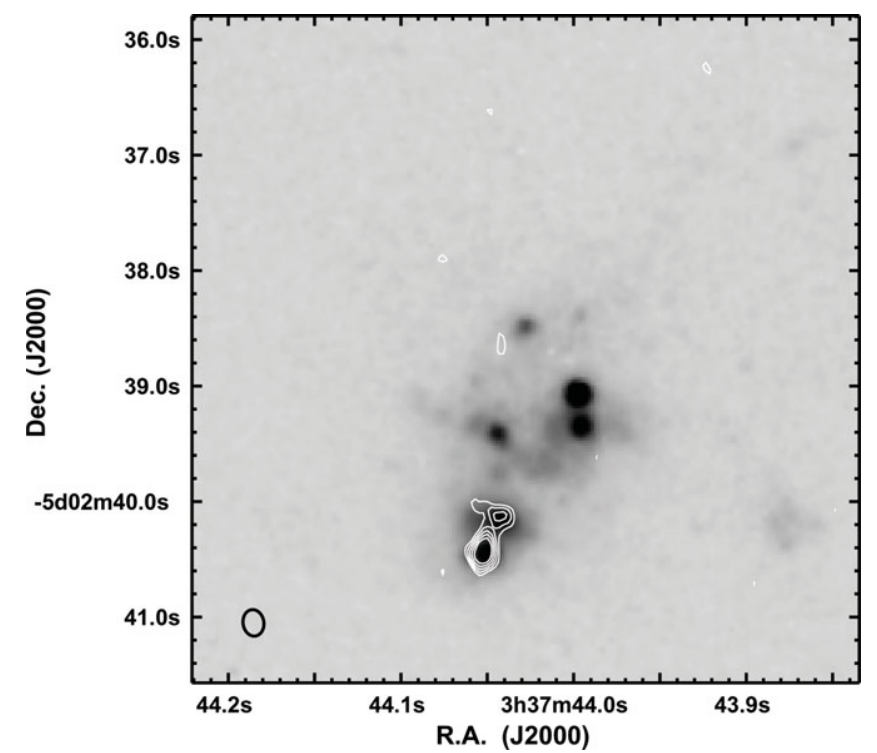

Figure 1. VLA $3.6 \mathrm{~cm}$ contours overlaid on an HST ACS F550M image of SBS 0335-053. The radio sources clearly correspond to previously known super star clusters in this galaxy. The synthesized beam is shown in the lower left corner.

of the star-forming regions is $\mathrm{n}_{\mathrm{e}} \gtrsim 10^{3}-10^{4} \mathrm{~cm}^{-3}$. However, given the evidence that the interstellar medium is inhomogeneous, peak densities are likely to reach significantly higher values.

\section{Concluding Remarks}

From this small sample of four galaxies with a detection rate of $25 \%$, one might be tempted to conclude that the conditions required for the formation of massive star clusters in low-metallicity dwarf galaxies are rare. Such a conclusion would present a problem for understanding globular cluster formation in the very early universe. First, the low rate of detections of thermal radio sources, at least in part, also reflects the very short time that clusters spend in the embedded phase; similar non-detection rates have been recovered from larger surveys not restricted to dwarf galaxies (Aversa et al. in prep); in other words, trying to catch these massive clusters while they are still embedded in their birth material virtually guarantees a low detection rate. Perhaps more importantly, we must also keep in mind that today's dwarf galaxies may not be the same beasts as the dwarf galaxies of $\sim 10$ Gyr ago. In particular, the density of galaxies was higher and interactions more frequent in the past. In this light, SBS 0335-052 is truly a gem in the relatively nearby universe for enabling this type of study, offering both very low metallicity and also extremely vigorous star formation. Unfortunately, at a distance of $\sim 55 \mathrm{Mpc}$, sensitivity and spatial resolution are already issues in obtaining and interpreting observations of SBS 0335-052, and this galaxy is the nearest currently known example of a such a lowmetallicity galaxy undergoing a starburst.

While this single galaxy SBS 0335-052 is a fascinating case study, it is important to keep an eye on the big picture. In this case, the main focus of this line of research is trying to constrain the physical conditions in the early universe that prodigiously gave rise to the ancient globular clusters we see today. There are a number of ways in which low metallicity might affect the formation and early evolution of massive star clusters, 
however the impact of metallicity in these intense star-forming environments remains an open issue. Both more extensive and more detailed studies are called for, and we are still a long way from that goal. However, one thing does seem quite clear; if the conditions are right, low-metallicity dwarf galaxies are certainly capable of producing globular clusters. The coming decade should bring about rapid progress in this area; the future is bright with a number of sensitive, high spatial resolution, and long wavelength observatories scheduled to become available over the next several years.

\section{References}

Bate, M. R. 2005, MSRAS, 363, 363

Beck, S. C., Turner, J. L., \& Kovo, O. 2000, AJ, 120, 244

Brodie, J. P. \& Strader, J. 2006, ARA\&A, 44, 193

Fall, S.M. \& Zhang, Q. 2001, ApJ, 561, 751

Harris, W. E. 1991, ARA\&A, 29, 543

Johnson, K.E. \& Kobulnicky, H.A. 2003, ApJ, 597, 923

Johnson, K. E., Indebetouw, R., \& Pisano, D. J. 2003, AJ, 126, 101

Johnson, K.E., Indebetouw, R.I., Watson, C., \& Kobulnicky, H.A. 2004, AJ, 128, 610

Reines, A.E., Johnson, K.E, \& Hunt, L.K. 2008, AJ, 136, 141

Schaerer, D. 2002, AAP, 382, 28

Smith, L.J., Norris, R.P.F., Crowther, P.A. 2002, MNRAS, 337, 1309

Tumlinson, J., Venkatesan, A., Shull, \& J.M. 2004, ApJ, 612, 602

Turner, J. L., Beck, S. C., \& Ho, P. T. P. 2000, ApJL, 532, L109

Vacca, W.D., Johnson, K.E., \& Conti, P.S. 2002 AJ, 123, 772

Whitmore, B. C. 2003, in A Decade of Hubble Space Telescope Science, 153

Whitmore, B. C., Chandar, R., \& Fall, S.M. 2007, 133, 1067 